\title{
PREVISÃO DE INSOLVÊNCIA: UM MODELO BASEADO EM ÍNDICES CONTÁBEIS COM UTILIZAÇÃO DA ANÁLISE DISCRIMINANTE*
}

\author{
Ailton Guimarães ${ }^{* *}$
}

\section{Tito Belchior Silva Moreira ${ }^{* * *}$}

\begin{abstract}
RESUMO O objetivo deste artigo é propor um modelo de previsão de insolvência baseado em indicadores contábeis com o uso da análise discriminante. Embora o assunto tenha sido bastante discutido, existe uma necessidade de aprimoramento dos modelos existentes, bem como da descoberta de novas variáveis preditoras e técnicas que melhor retratem o comportamento das empresas sob a ótica do risco de crédito. Com relação às variáveis preditoras da função discriminante, gerada pelo método passo a passo, o trabalho confirmou o poder discriminatório daquelas que evidenciam as decisões financeiras sobre estrutura de ativos, estrutura de capital e geração de caixa. Outro resultado importante diz respeito ao poder discriminatório da variável representativa do montante dos recursos próprios disponíveis. Essa variável, que é utilizada em estudos sobre solvência de instituições financeiras, apresentou resultados promissores também para empresas não financeiras. $\mathrm{O}$ modelo proposto, estatisticamente significante, representa, através das suas variáveis independentes, o resultado das decisões das firmas com bom índice de acerto nas predições sobre insolvência.
\end{abstract}

Palavras-chave: insolvência; variáveis preditoras; poder discriminatório Código JEL: C1, G33

\footnotetext{
* Artigo recebido em 4 de outubro de 2006 e aprovado em 28 de fevereiro de 2008. Os autores agradecem os pertinentes comentários de Daniel Cajueiro e dos pareceristas anônimos. Este artigo reflete as opiniões dos autores. O Banco Central do Brasil não se responsabiliza e nem pode ser responsabilizado por prejuízos de qualquer natureza em decorrência do uso das informações deste artigo.

** Analista do Banco Central e mestre em Economia pela UCB, e-mail: ailton.guimaraes@bcb.gov.br

*** Professor-doutor do Departamento de Economia da UCB, e-mail: tito@pos.ucb.br
} 


\title{
INSOLVENCY PREDICTOR: A MODEL BASED ON ACCOUNT INDEX WITH APPLICATION OF DISCRIMINANT ANALYSIS
}

\begin{abstract}
The objective of this paper is to propose an insolvency forecast model based on accounting indicators using discriminant analysis. Although this issue had been thoroughly discussed, there is a need of the existing models enhancement as well as the uncovering of new forecasting variables and techniques that would better describe the behavior of corporation through the credit risk prospective. Regarding the predictive variables of the discriminant function, generated by the stepwise method, the research confirmed the discriminatory power of those variables, which emphasize the financial decisions over asset structure, capital structure and the cash flow related variable. Another important result is related to the discriminatory power of the variable representing the amount of equity. The variable, which is used on studies related to financial institutions solvency, showed also promising results for non-financial corporations. The proposed model, which is statistically significant, represents the result of company's decisions showing a good level of prediction on insolvency forecast, through its independent variables.
\end{abstract}

Key words: insolvency; predictive variables; discriminatory power 


\section{INTRODUÇÃO}

Com a recente desregulamentação global e a crescente mobilidade do capital — conseqüência das alterações políticas, tecnológicas e, principalmente, econômicas ocorridas em todo o mundo - , a oferta de recursos e a demanda por novos produtos financeiros para captação tiveram forte crescimento, assim como o interesse pelo desenvolvimento da modelagem do risco de crédito.

No Brasil, a estabilidade econômica, a partir da vigência do Plano Real em 1994, provocou aumento vertiginoso no volume de operações de crédito entre empresas não financeiras. Como conseqüência, o desenvolvimento e a utilização de modelos de classificação do risco de crédito, objetivando minimizar possíveis perdas de fluxos de caixa e do valor presente das firmas, também experimentaram forte evolução.

A literatura correlata apresenta grande quantidade de publicações que descrevem diferentes técnicas e modelos de avaliação do risco de crédito. Esses modelos são, em geral, divididos em dois grupos: os modelos quantitativos sustentados por métodos estatísticos, que têm como base características das carteiras ou dados específicos de clientes, e os modelos qualitativos, construídos a partir de informações dos clientes ou fontes externas (centrais de riscos, agências de rating ${ }^{1}$ etc.).

O modelo proposto neste trabalho é do tipo quantitativo, com utilização da análise discriminante multivariada, tendo como base indicadores contábeis de empresas. O problema a ser resolvido é a estimação de uma função que melhor discrimine empresas solventes e empresas insolventes.

Embora os modelos multivariados, principalmente aqueles baseados em dados contábeis, sejam criticados sob a alegação de que são essencialmente empíricos e pela não-consideração de variáveis ou riscos fora dos balanços, diversos estudos mostraram que eles têm apresentado bons índices de acertos nas predições ao longo de muitos períodos, como demonstrado na tabela 1 .

Os registros contábeis refletem as ações dos gestores das firmas e essas ações devem ser orientadas pela racionalidade econômica que, segundo a teoria da firma, é a maximização do lucro ou da riqueza do acionista. Maxi- 
Tabela 1: Percentual de acerto de modelos construídos com análise discriminante

\begin{tabular}{|c|c|c|c|c|c|c|}
\hline \multirow[t]{2}{*}{ Autor } & \multirow{2}{*}{$\begin{array}{c}\text { Ano do } \\
\text { desenvolvimento } \\
\text { do modelo }\end{array}$} & \multicolumn{2}{|c|}{$\begin{array}{c}\text { Quantidade de } \\
\text { empresas analisadas }\end{array}$} & \multirow[t]{2}{*}{ Setor } & \multirow{2}{*}{$\begin{array}{c}\text { Anos } \\
\text { antes da } \\
\text { quebra }\end{array}$} & \multirow{2}{*}{$\begin{array}{l}\text { Percentual } \\
\text { de acerto } \\
(\%)\end{array}$} \\
\hline & & Boas & Ruins & & & \\
\hline Altman & 1968 & 33 & 33 & Diversos & 1 & 93,9 \\
\hline Elizabetsky & 1976 & 274 & 99 & Confecções & 1 & 69 \\
\hline Matias & 1978 & 50 & 50 & Diversos & 1 & 74 \\
\hline Altman & 1979 & 35 & 23 & Diversos & 1 & 80 \\
\hline Pereira & 1982 & 194 & 61 & Indústria & 1 & 74 \\
\hline Pereira & 1982 & 40 & 18 & Comércio & 1 & 79 \\
\hline Barth & 2003 & 726 & 726 & Diversos & 1 & 72 \\
\hline
\end{tabular}

Fontes: SILVA, José Pereira. Gestão e análise de risco de crédito. São Paulo: Atlas, 2003.

BARTH, Nelson Lerner. Inadimplência: construção de modelos de previsão. São Paulo: Nobel, 2004.

CAOUETTE et al. Gestão do risco de crédito: próximo grande desafio financeiro.

mizar a riqueza do acionista significa alocar recursos em ativos cujos riscos sejam compatíveis com seus retornos ou escolher a estrutura ótima de capital ou, ainda, minimizar custos. Todas estas ações podem ser extraídas de indicadores contábeis.

Na primeira parte do artigo, a introdução, foi feita a justificativa do estudo do tema, qual o problema associado ao tema e a importância da utilização de indicadores contábeis na construção de modelos de previsão de default. ${ }^{2} \mathrm{Na}$ segunda, é apresentada a metodologia utilizada com a descrição dos dados e a técnica estatística do modelo. A terceira parte diz respeito à análise dos resultados e em seguida temos a conclusão do trabalho.

\section{METODOLOGIA}

\subsection{A amostra utilizada}

A amostra utilizada neste trabalho é composta de informações econômico -financeiras de 116 empresas de capital aberto de 17 setores diferentes, no período 1994 a 2003, coletadas do banco de dados do Sistema de Análise de Balanços de Empresas - SABE, construído pelo Instituto Brasileiro do Mercado de Capitais - IBMEC. Dentro desse universo, as empresas que se encontravam no estado de insolvência, definido no item seguinte, foram selecionadas como ruins. Os indicadores contábeis das empresas ruins foram extraídos dos demonstrativos contábeis referentes a um ano antes da entra- 
da no estado de insolvência e as informações das empresas boas, do mesmo setor, na mesma data. Assim, se uma empresa entrou no estado de insolvência em 1996, foram extraídos, para este estudo, os dados do seu balanço patrimonial de 1995 e para efeito de comparação foram utilizados os dados de uma empresa boa, que não entrou no estado de insolvência, do mesmo setor, da mesma data, 1995.

A partir dessa primeira seleção foram formadas amostras de dois tipos:

(1) Uma amostra de desenvolvimento, composta de 35 empresas que entraram no estado de insolvência, empresas ruins, e igual número de empresas que não entraram no estado de insolvência, empresas boas, do mesmo setor ou com ativos equivalentes. Esse tipo de amostra, denominado amostra emparelhada, é constituído pelos elementos que possuem fatores ou fontes de variabilidade (no caso, o setor ou volume de ativos) que nada têm a ver, diretamente, com o estudo, mas que podem interferir nos resultados esperados; $\mathrm{e}$

(2) Uma amostra de validação ou controle, contendo 23 empresas ruins e igual número de empresas boas, selecionadas pelos mesmos critérios da amostra de desenvolvimento.

\subsection{A análise discriminante}

Para Hair Jr. et al. (2005), a análise discriminante múltipla é a técnica multivariada adequada para estudar problemas em que a variável estatística (combinação linear de variáveis com pesos determinados empiricamente) é dicotômica e, portanto, não métrica. Então, ela é indicada para construir modelos de previsão de inadimplência, cujo objetivo principal é a classificação de um aspirante a crédito em um determinado grupo de:

(a) Provável adimplente (solvente) ou;

(b) Provável inadimplente (insolvente).

A técnica procura, basicamente, responder se um elemento pertence ou não a uma determinada categoria. Tratando-se da análise de risco de crédito, a análise discriminante, ou especificamente a função discriminante, indicará se uma empresa pertence a um grupo de firmas solventes ou ao conjunto das insolventes.

O valor previsto da função da discriminante é o escore discriminante, o qual é calculado para cada objeto (pessoa, empresa ou produto) na análise. 
A função discriminante tem a seguinte forma:

$$
Z=a+W_{1} \cdot X_{1}+W_{2} \cdot X_{2}+W_{3} \cdot X_{3}+\ldots+W_{n} \cdot X_{n}
$$

onde:

$Z=$ escore discriminante ou categoria

$a=$ intercepto

$W_{i}=$ peso ou coeficiente discriminante para a variável i, com $1=1,2$, $3, \ldots n$

$X_{i}=$ variável independente $\mathrm{i}$

As variáveis independentes relevantes, formadoras da função discriminante, são obtidas por recurso à estatística lambda $(\lambda)$ de Wilks.

A estatística para cada uma das variáveis, segundo Maroco (2003), é dada por:

$$
\lambda=\frac{S Q E}{S Q T}
$$

sendo:

(a) $S Q E=\sum(y-\bar{y})^{2}$ soma dos quadrados dos erros dentro dos grupos

(b) $S Q T=\sum(y-\hat{y})^{2}=$ soma dos quadrados totais

(c) $y=$ variável sob estudo

(d) $\bar{y}=$ valor médio de $\mathrm{y}$

(e) $\hat{y}=$ valor estimado de $y$

Os pesos ou coeficientes $w_{1}, w_{2}, \ldots . ., w_{n}$ são estimados de modo que a variabilidade dos escores $\left(D_{i}\right)$ da função discriminante seja máxima entre os grupos e mínima dentro dos grupos. Eles representam uma medida relativa da importância das variáveis originais na função estimada. Quanto maior for o coeficiente das variáveis independentes, maior será a sua contribuição na discriminação entre os grupos, passo importante para interpretação e análise do modelo.

Dessa forma, dados $p$-variáveis e $g$-grupos é possível estabelecer $m=\min$ $(g-1 ; p)$ funções discriminantes que são combinações lineares das $p$-variáveis, tal que: 


$$
D_{i}=w_{1} X_{1}+w_{2} X_{2}+\ldots \ldots .+w_{n} X_{n}, \operatorname{com} i=1,2, \ldots \ldots, n
$$

O nível de significância da função é estimado com base em uma transformação qui-quadrado da estatística $(\lambda)$ de Wilks.

A classificação de indivíduos em classes utilizadas para estimar a função discriminante é procedida, de modo a que cada indivíduo seja inserido no grupo cujo centróide (valor médio para os escores discriminantes de todos os elementos, em uma dada categoria ou grupo) se encontra mais próximo.

O ponto de corte ou zona de fronteira é dado por:

$$
f=\frac{n_{0} \bar{d}_{0}+n_{1} \bar{d}_{1}}{n_{0}+n_{1}}
$$

Onde:

$\bar{d}_{0}$ e $\overline{d_{1}}$ são as médias (centróides) da função discriminante nos grupos 0 e 1 e $n_{0}$ e $n_{1}$ são as dimensões (números de indivíduos) desses grupos.

Assim, um determinado indivíduo pertencerá ao grupo 1 (empresas solventes) se o seu escore, $D_{i}$, for maior que 0 . Um resultado diferente irá classificá-lo no grupo 0 (empresas insolventes).

\subsubsection{Estudos sobre análise discriminante}

Até 1980, os principais modelos para previsão de inadimplência eram baseados na técnica de análise discriminante. Em 1980, surgiram os modelos baseados na análise logística e logo após outras técnicas foram empregadas no desenvolvimento de modelos de previsão de falência, como as técnicas de redes neurais e algoritmos genéticos.

Back et al. (1996), utilizando dados contábeis de firmas finlandesas do período 1986 a 1989, aplicaram e compararam modelos baseados nestas técnicas e chegaram aos resultados apresentados na tabela 2.

Barth (2004), usando uma mostra de 1.452 empresas brasileiras, comparou o desempenho de métodos baseados em análise discriminante, regressão logística, algoritmos genéticos e redes neurais. O desempenho obtido com análise discriminante foi superior ao obtido com algoritmo genético (ambos geradores de funções discriminantes lineares), e os acertos conseguidos com o uso de redes neurais foram superiores aos da regressão logística. 
Tabela 2: Desempenho dos principais métodos utilizados nos modelos de previsão de insolvência

\begin{tabular}{lcccccccccc}
\hline & \multicolumn{3}{c}{ Erro tipo I } & \multicolumn{3}{c}{ Erro tipo II } & \multicolumn{3}{c}{ Erro total } \\
\hline $\begin{array}{l}\text { Anos } \\
\text { antes } \\
\text { da quebra }\end{array}$ & $\begin{array}{c}\text { Análise } \\
\text { discriminante } \\
(\%)\end{array}$ & $\begin{array}{c}\text { Análise } \\
\text { logística } \\
(\%)\end{array}$ & $\begin{array}{c}\text { Redes } \\
\text { neurais } \\
(\%)\end{array}$ & $\begin{array}{c}\text { Análise } \\
\text { discriminante } \\
(\%)\end{array}$ & $\begin{array}{c}\text { Análise } \\
\text { logística } \\
(\%)\end{array}$ & $\begin{array}{c}\text { Redes } \\
\text { neurais } \\
(\%)\end{array}$ & $\begin{array}{c}\text { Análise } \\
\text { discriminante } \\
(\%)\end{array}$ & $\begin{array}{c}\text { Análise } \\
\text { logística } \\
(\%)\end{array}$ & $\begin{array}{c}\text { Redes } \\
\text { neurais } \\
(\%)\end{array}$ \\
\hline 1 & 13,51 & 13,51 & 5,26 & 16,22 & 13,51 & 0,00 & 14,86 & 3,51 & 2,70 \\
\hline 2 & 24,32 & 27,03 & 26,32 & 18,92 & 29,73 & 27,78 & 21,62 & 28,40 & 27,03 \\
\hline 3 & 16,22 & 16,22 & 5,26 & 37,84 & 35,14 & 27,78 & 27,03 & 25,70 & 16,22 \\
\hline Fonte: Elaborada pelos autores.
\end{tabular}

$\mathrm{Na}$ comparação entre os quatro modelos, levando-se em consideração o maior percentual de acerto, o modelo baseado em redes neurais mostrou-se mais adequado. Porém, se a interpretação do modelo for considerada um quesito importante para a escolha, a análise discriminante linear será mais apropriada. Esse fato mostra que um modelo que tem por objeto a discriminação entre grupos não pode ser escolhido somente com base em um único critério, pois os modelos têm vantagens e desvantagens que devem ser avaliadas na decisão. No estudo de Barth, foram levados em consideração, na comparação dos modelos, o percentual de acerto, o tempo de processamento e a facilidade de interpretação.

Lo (1986) produziu um trabalho no qual comparou a análise discriminante com a análise logística e concluiu, após um teste de especificação para a análise de falência de corporações, que a hipótese de equivalência entre a análise discriminante e logit não pode ser rejeitada. $\mathrm{O}$ resultado do trabalho indicou, ainda, que, para fins de classificação, a não-normalidade pode ser menos problemática do que sugeriram estudos anteriores.

\subsubsection{Suposições da análise discriminante}

Para Hair Jr. et al. (2005), certas condições são requeridas para a aplicação da análise discriminante. As suposições mais importantes são a de normalidade das variáveis independentes e igualdade nas matrizes de dispersão e covariância dos grupos. A falta de normalidade pode causar problemas na estimação da função discriminante e matrizes de covariâncias desiguais podem afetar negativamente o processo de classificação.

Uma outra característica dos dados que pode comprometer os resultados é a multicolinearidade entre variáveis independentes. Essa característica indica se duas ou mais variáveis são correlacionadas, uma variável pode ser 
explicada por outra sem acréscimo significativo ao poder elucidativo do conjunto como um todo.

O pressuposto de linearidade das relações entre as variáveis está implícito na função discriminante, pois relações não lineares não são refletidas na função, a menos que transformações específicas de variáveis sejam executadas para representar efeitos não lineares.

\subsection{0 evento a ser modelado}

O evento a ser modelado neste trabalho é o estado de insolvência ou iliquidez, declarado ou presumido, um ano antes da sua ocorrência.

Insolvência, na visão jurídica, é a situação em que o ativo do devedor é insuficiente para fazer face ao passivo, isto é, quando há um excedente de passivo em relação ao seu ativo patrimonial.

Nos meios comerciais e financeiros a insolvência significa falência ou concordata. Essas situações, concordata ou falência, são expressamente declaradas pela própria empresa ou por um credor. No entanto, existem situações, não raras, exteriorizadas nos demonstrativos contábeis, em que a empresa encontra-se no estado de insolvência, mas não a declara. Nesse sentido, vale conhecer os ensinamentos do ilustre professor Rubens Requião (1998), em sua obra Curso de direito falimentar, em que afirma:

A insolvência, conceitualmente, é um fato que, geralmente, se infere da insuficiência do patrimônio do devedor para o pagamento de suas dívidas, sendo que esse estado tanto pode ser confessado pelo empresário comercial, como pode ser presumido por atos que exteriorizem a ruína da empresa.

Então, o estado de insolvência pode ser declarado, casos da concordata e falência, ou presumido, situação em que os demonstrativos contábeis mostram claramente que o total dos ativos é menor que o valor das dívidas, como demonstrado a seguir.

Sejam $A_{t}, D_{t}$ e $\mathrm{PL}_{t}$, respectivamente, o valor total dos ativos de uma empresa, o valor das dívidas e o valor do capital próprio no tempo $t$. Se:

(i) $A_{t}=D_{t}+\mathrm{PL}_{t}$, com $D_{t} \geq 0$ e $\mathrm{PL}_{t} \geq 0$, então a empresa é solvente, pois $A_{t} \geq D_{\dot{t}}$

(ii) $A_{t}=D_{t}+\mathrm{PL}_{t}$, com $D_{t} \geq 0$ e $\mathrm{PL}_{t}<0$, então a empresa está insolvente, pois $A_{t}<D_{t}$. 
O objetivo deste estudo é modelar o comportamento das empresas antes da entrada no estado de insolvência, declarado (concordata ou falência) ou presumido (passivos > ativos) e, a partir daí, construir um modelo que possibilite a previsão desse evento.

\subsection{Seleção das Variáveis para estudo}

Sanvicente e Minardi (1998) desenvolveram trabalho exploratório tendo como objetivo principal a identificação de índices contábeis mais significativos para prever concordatas de empresas no Brasil e como objetivo secundário testar a crença do mercado de que demonstrações contábeis não fornecem boa informação para análise de crédito de empresas. Com o uso da análise discriminante, os autores concluíram que os indicadores contábeis com maior poder de previsão de insolvência foram os índices de liquidez, seguidos por indicadores de lucros retidos, rentabilidade, endividamento e cobertura de juros que aumentaram o poder de previsão do modelo.

Caouette, Altman e Narayanam (1999) enfatizam a importância do fluxo de caixa na definição da liquidez das empresas, principalmente o fluxo de caixa operacional, que mostra se as empresas são capazes de gerar recursos suficientes a partir de suas operações principais, e acrescentam que, de modo geral, os índices de lucratividade, liquidez e endividamento parecem ser os mais apropriados à construção dos modelos de previsão de insolvência ancorados em dados contábeis.

Harris e Raviv (1991), em seu estudo teoria da estrutura de capital, concluíram, baseados em resultados empíricos, que o nível de endividamento, o valor da empresa e a probabilidade de insolvência têm correlação positiva.

Os resultados dos estudos aqui mencionados permitem inferir que a decisão sobre alocação de recursos tem importante função na criação de valor e no nível da capacidade de pagamento. A estrutura de capital, financiamento ou endividamento tem implicações sobre o custo de capital, resultados e, também, sobre a capacidade de pagamento. Através dos resultados - lucros ou prejuízos - , podemos medir a rentabilidade auferida e os fluxos de caixa necessários para suprir os compromissos com terceiros, investimentos e remuneração dos acionistas. Então, a escolha das variáveis do presente estudo 
será baseada nesses resultados e, utilizando as técnicas estatísticas apropriadas, serão determinadas aquelas com maior poder de explicação sobre o evento a ser modelado.

Como ponto de partida, as variáveis foram segregadas conforme suas referências com as decisões financeiras ou subgrupos contábeis como apresentadas a seguir.

\subsubsection{Variáveis relacionadas à estrutura de ativos}

A decisão sobre a alocação de recursos tem importantes reflexos sobre a geração de caixa da firma. Os ativos devem atender, principalmente, ao equilíbrio da relação risco e retorno, mas devem também proporcionar ao gestor boa capacidade de liquidez. Os indicadores a seguir evidenciam a escolha da firma com relação à decisão de alocação do total dos recursos captados.

(i) $E s t A=\frac{\text { Capital de Giro Líquido }}{\text { Ativo Total }}$, Capital de Giro Líquido em relação ao ativo total. Variável representativa da composição dos ativos. O capital de giro líquido é definido pela diferença entre os ativos com maior liquidez (ativos circulantes) e as dívidas de curto prazo (passivo circulante). Quanto maior o resultado dessa diferença, melhor será a capacidade de pagamento. Vale lembrar que, assim como o montante de recursos alocados em ativos de curto prazo, a qualidade desses bens e direitos é fundamental, pois a ocorrência de perdas implicará comprometimento da capacidade de pagamento de curto prazo.

(ii) AGRen $=$ (Ativo total - Ativos não geradores de renda), Ativos geradores de renda em relação ao ativo total. Variável que evidencia o montante de recursos alocados em ativos que têm relação direta com a geração de receitas da empresa. Quanto mais recursos forem direcionados para ativos geradores de renda, melhor a capacidade de geração de caixa.

(iii) ACir = Tamanho do Ativo Circulante. Variável indicativa da capacidade de pagamento de curto prazo. 


\subsubsection{Variáveis relacionadas à estrutura de capital ou de financiamento}

A composição das fontes de financiamento ou estrutura de capital é de extrema importância para os resultados e continuidade da companhia. Quanto maior o montante de recursos captados junto a terceiros, maior o risco de bancarrota e, sendo assim, o gerente é pressionado a ser cada vez mais eficiente na busca da obtenção de fluxos de caixa suficientes para garantir o pagamento dos compromissos financeiros e a remuneração dos acionistas. Os índices a seguir evidenciam a escolha da firma com relação à decisão de captação dos recursos e o risco associado a essa decisão.

(iv) $E s t C=\frac{\text { Passivo Circulante }+ \text { Exigível a Longo Prazo }}{\text { Patrimônio Líquido }}$.

Variável derivada da estrutura de capital. Mostra a proporção entre o montante de recursos de terceiros (soma do passivo circulante mais o exigível a longo prazo) em relação aos recursos próprios (patrimônio líquido). Considerando que os recursos próprios investidos na firma são, em última instância, a garantia para liquidação dos compromissos com terceiros, quanto maior o resultado dessa relação, maior o risco do negócio.

(v) $M C P d=(P L-$ Ativos de Baixa Liquidez $)$.

Montante dos recursos próprios disponíveis. Variável que procura evidenciar o montante do capital próprio disponível. Computada pela diferença entre o montante de recursos próprios e os valores alocados em ativos de baixa liquidez, tais como despesas pagas antecipadamente, impostos a recuperar, ativo diferido, incentivos fiscais e empréstimos compulsórios. O montante de recursos próprios tem importante função de sinalização quanto à capacidade de absorver perdas e, sendo assim, quanto menor o comprometimento do capital próprio com ativos de baixa liquidez melhor a garantia dos compromissos com terceiros.

(vi) $A F R L P=$ Ativo Total - Passivo Circulante.

Ativos financiados com recursos de longo prazo. Quanto maior o volume de ativos financiados com obrigações de longo prazo, melhor a capacidade de pagamento de curto prazo. 


\subsubsection{Variáveis relacionadas à geração de caixa ou retornos}

A capacidade de geração de caixa é um indicativo do valor da firma e da qualidade das decisões sobre captação e aplicação dos recursos. As empresas com boa capacidade de geração de caixa são, em geral, mais sólidas. As variáveis a seguir mostram o nível de geração de caixa e de retorno.

(vii) $\quad$ EBITCT $=\frac{\text { EBIT }}{\text { PC }+ \text { ELP }}$.

Variável que mede a proporção do fluxo de caixa da empresa (Resultado Operacional - Resultado Financeiro + Depreciação e Amortização - Resultado da Equivalência Patrimonial) em relação a suas despesas financeiras. O EBIT, Earnings Before Interest and Tax, é uma medida do fluxo de caixa operacional das firmas. Quanto maior o resultado desse quociente, menor a probabilidade de a empresa apresentar dificuldade na liquidação de seus compromissos financeiros.

(viii) $\operatorname{ReCP}=\frac{\text { Lucro Líquido }}{\text { Patrimônio Líquido/Índice de Inflação }}$.

Retorno do capital próprio - demonstra quão eficiente foi a gestão dos recursos próprios investidos no negócio. O retorno do capital próprio depende da eficiência operacional (margem de lucro), da gestão dos ativos (giro dos ativos) e da alavancagem financeira.

(ix) $R O R L=\frac{\text { Lucro Operacional }- \text { Despesas Financeiras }}{\text { Receitas Líquidas }}$.

Variável representativa da eficiência operacional. Procura evidenciar qual o retorno dos ativos após o pagamento dos custos fixos e variáveis e dos encargos incidentes sobre os recursos de terceiros. As empresas que conseguem alocar os recursos de terceiros em ativos com rentabilidades superiores aos custos dessas fontes auferem maiores retornos operacionais.

(x) ROACir $=\frac{\text { Receita Operacional Líquida }}{\text { Ativo Circulante }}$. 
Variável que evidencia a proporção entre vendas e ativos de curto prazo. Em geral, variações nas vendas refletem-se no volume de recursos aplicados no ativo circulante, que, por sua vez, precisam de fontes adequadas de financiamento.

\subsubsection{Variáveis relacionadas à liquidez}

A capacidade de cumprir obrigações financeiras, principalmente as de curto prazo, é fundamental para a continuidade da empresa. A decisão sobre a alocação de recursos determina também o nível de liquidez da firma. A variável a seguir mostra o nível de liquidez das firmas.

(xi) Liq $=\frac{\text { Ativo Circulante }}{\text { Ativo Total }}-\frac{\text { Passivo Circulante }}{\text { Passivo Total }}$.

Medida de liquidez das empresas. Quanto maior a proporção de recursos alocados em ativos de curto prazo comparados com as exigibilidades de curto prazo, melhor será a capacidade de pagamento da firma.

\subsubsection{Variáveis relacionadas à eficiência operacional}

A eficiência operacional das empresas depende significativamente das decisões sobre captação e alocação de recursos e também da performance do gerente no que diz respeito aos custos de produção. A eficiência operacional contribui para aumentar a geração de caixa e a criação de valor. $O$ indicador a seguir é utilizado como medida para medir a eficiência das firmas.

(xii) $\quad E V A R L=\frac{\text { EVA }}{\text { Receita Líquida }}$.

Variável que mostra quanto valor é adicionado à riqueza do acionista em relação a suas vendas. O EVA, Economic Value Added ou Valor Econômico Agregado, é fundamentado no pressuposto econômico de que as empresas, para serem viáveis, devem gerar mais riqueza que o custo de capital que empregam. Um aumento sustentado no EVA resultará em um aumento no valor de mercado de uma companhia. 
Quadro 1: Transformações de variáveis

\begin{tabular}{|c|c|}
\hline Variável original & Nova variável \\
\hline AGRen $=($ Ativo Total - Ativos & LogAGRen = Logaritmo (Ativo Total - Ativos Não \\
\hline Não Geradores de Renda) & Geradores de Renda) \\
\hline ACir = Ativo Circulante & LogACir = Logaritmo (Ativo Circulante) \\
\hline EstC $=($ Passivo Circulante + Exigível a & EstC $=($ Passivo Circulante + Exigível a Longo \\
\hline$\underline{\text { Longo Prazo)/(Passivo Total) }}$ & Prazo)/(Passivo Total) $)^{2}$ \\
\hline $\mathrm{MCPd}=$ (Patrimônio Líquido - Ativos de & RCMCPd = (Patrimônio Líquido - Ativos de Baixa \\
\hline Baixa Liquidez) & Liquidez) $)^{1 / 3}$ \\
\hline \multirow[t]{2}{*}{ AFRLP $=($ Ativo Total - Passivo Circulante $)$} & LogAFRLP = Logaritmo (Ativo Total - Passivo \\
\hline & Circulante) \\
\hline ROACir = (Receita Operacional/Ativo Circulante) & QROACir $=(\text { Receita Operacional/Ativo Circulante })^{2}$ \\
\hline
\end{tabular}

\subsection{Transformação de variáveis}

O ponto de partida para o entendimento da natureza de qualquer variável é o estudo das suas características. Algumas medidas estatísticas podem ser utilizadas para verificar qual o tipo de distribuição, a relação entre as variáveis. É importante, também, examinar se as diferenças entre os grupos são suficientes para suportar a significância estatística.

A verificação de ocorrência de valores extremos também é importante, assim como a verificação da distribuição de probabilidade das variáveis independentes. Hair et al. (2005) sugere alguns procedimentos para correção de problemas de normalidade, homocedasticidade e linearidade. De acordo com estes procedimentos, algumas transformações foram aplicadas às variáveis em estudo, como resumido no quadro 1.

\subsection{Análise exploratória de dados}

Em seguida às transformações, foram gerados gráficos do tipo boxplot para exame das características das possíveis variáveis preditoras. Esse procedimento, denominado análise exploratória dos dados, é recomendável para que o uso das ferramentas estatísticas tenha resultados satisfatórios.

Os gráficos têm um grande poder de mostrar padrões de dados sob estudo, e o boxplot, do tipo histograma, fornece informações sobre esses padrões, mostrando as seguintes características do conjunto de dados: dispersão, assimetria e valores discrepantes (outliers). 
Tabela 3: Escala de significância de Fisher

\begin{tabular}{lcccccc}
\hline$p$-valor & 0,100 & 0,050 & 0,025 & 0,010 & 0,005 & 0,001 \\
\hline$(10 \%)$ & $(5 \%)$ & $(2,5 \%)$ & $(1 \%)$ & $(0,5 \%)$ & $(0,1 \%)$ \\
\hline $\begin{array}{l}\text { Evidência } \\
\text { contra Ho }\end{array}$ & Marginal & Moderada & Substancial & Forte & Muito forte & Fortíssima \\
\hline Fonte: BUSSAB; MORETIN. Estatística básica. São Paulo: Saraiva, 2003. & & &
\end{tabular}

\subsection{Nível de significância}

Antes da aplicação de testes estatísticos devemos estabelecer o nível de significância, normalmente denominado $\alpha$. Ele representa uma medida ou grau de certeza a partir do qual assumimos como real o resultado da estatística obtido no estudo ou, em outras palavras, a probabilidade de que uma prova estatística apresente um valor ( $p$-valor) que conduza à rejeição da hipótese nula, $H_{0}$, quando esta é verdadeira. A regra geral é rejeitar $H_{0}$, se o $p$-valor $\leq \alpha$.

Bussab e Morettin (2003) apresentam a tabela 3 que relaciona o nível de significância com as evidências contra a hipótese nula.

A escala mostra que, quanto menor o $p$-valor, mais forte será a evidência contra $H_{0}$.

Outros parâmetros, como controle das variáveis, confiabilidade dos dados, características das variáveis da amostra e fidedignidade dos instrumentos de medidas, também devem ser levados em consideração na escolha do nível de significância. Para este estudo, foi definido o nível de significância de $5 \%$.

\subsection{Teste de normalidade}

Para Sharma (1996), testes de normalidade multivariada são muito complexos e difíceis e o entendimento destes pode ser facilitado pelos testes de normalidade univariada. Acrescente-se que, embora seja possível termos distribuição multivariada não normal quando as distribuições marginais são normais, esses casos são raros.

Para verificar o tipo de distribuição das variáveis independentes, normalidade univariada, utilizaremos o teste de Kolmogorov-Smirnov (K-S), e para testar a normalidade multivariada usaremos os testes Mardia-Assimetria, Mardia-Curtose e Henze-Zirkler. A robustez dos testes aqui utilizados foi verificada por Mecklin e Mundfrom (2003). Eles trabalharam com uma amostra de 10 mil dados gerados através de Simulação de Monte Carlo para 
avaliar oito procedimentos normalmente usados para detectar normalidade multivariada, e concluíram ser o teste Henze-Zirkler o mais apropriado para esse fim. Os testes Mardia-Assimetria e Mardia-Curtose devem ser usados como medidas complementares, principalmente quando o teste Henze-Zirkler não for capaz de diagnosticar a não-normalidade.

Para computar os testes de normalidade multivariada, utilizaremos o software estatístico Systat, versão 12, desenvolvido pela Systat Software Inc. (SSI).

A hipótese nula para todos esses testes é a de que os resíduos são normalmente distribuídos.

\subsection{Teste de homogeneidade das matrizes de variância-covariância}

O pressuposto de que a matriz de variância-covariância para cada um dos grupos em estudo, em relação a cada uma das $p$-variáveis, provenha de uma mesma população de variância-covariância, de modo que uma estimativa conjunta da variância residual possa ser obtida, não pode ser violado, sob pena de comprometimento da robustez da função discriminante. O teste mais utilizado para avaliar a hipótese de homogeneidade das matrizes de variância-covariância é o $M$ de Box. As hipóteses do teste são:

$$
\begin{aligned}
& H_{0}: \sum_{1}=\sum_{2}=\ldots \ldots . .=\sum_{m} \\
& H_{1}: \exists i, \mathrm{j}: \sum_{i}=\sum_{j} \operatorname{com~i} \neq \mathrm{j} ; \mathrm{i}, \mathrm{j}=\{1, \ldots \ldots, \mathrm{m}\}
\end{aligned}
$$

Se a dimensão de todas as amostras for igual, a função discriminante pode ser considerada robusta relativamente à não-violação do pressuposto de homogeneidade das matrizes de variância-covariância.

\subsection{Seleção das variáveis discriminantes}

A seleção das variáveis discriminantes $(m)$, dado um conjunto de $p$-variáveis, é o passo inicial para estimação da função discriminante.

$\mathrm{Na}$ análise discriminante, a decisão da utilização de determinada variável depende do resultado do teste de igualdade de suas médias nos grupos em estudo através das hipóteses:

$H_{0}: \mu_{1 i}=\mu_{2 i}=\ldots=\mu_{k i}$, a variável apresenta média semelhante nos grupos, ou seja, não é discriminante.

$H_{1}: \exists \mu_{1 i} \neq \mu_{k i}$, as médias da variável em estudo são diferentes. 
O SPSS apresenta o $p$-valor — o menor nível de significância $(\alpha)$, a partir do qual rejeitamos a hipótese nula, $H_{0}$, associado à estatística $\mathrm{F}$. Se $p$-valor $>\alpha$, devemos aceitar $H_{0}$; caso contrário, devemos rejeitar essa hipótese em favor da hipótese alternativa, concluindo que a variável é significante e discrimina os grupos, pois $p$-valor $\leq \alpha$.

\subsection{Estimação da função discriminante}

Dois métodos computacionais podem ser utilizados para determinar uma função discriminante: o método simultâneo ou direto e o método stepwise ou passo a passo.

Na estimação simultânea, a função discriminante é computada de modo que todas as variáveis independentes sejam consideradas conjuntamente, sem considerar o poder discriminatório de cada uma delas. Assim, a função discriminante é computada com base no conjunto inteiro de variáveis independentes, sem consideração do poder discriminatório de cada uma dessas variáveis.

No método passo a passo, as variáveis são escolhidas conforme seu poder discriminatório. Primeiramente, é escolhida aquela variável que melhor discrimina os grupos, utilizando para isso, geralmente, o lambda de Wilks e estatística F. Em seguida, ela é combinada com cada uma das outras variáveis, para que seja selecionado o par de variáveis que melhora o poder discriminatório. $\mathrm{O}$ passo seguinte é combinar esse par com as variáveis restantes, de modo que seja encontrada outra variável que melhore ainda mais o poder discriminatório. Esse procedimento é repetido até que seja encontrado o melhor conjunto de variáveis para compor a função discriminante. O método permite, no caso de um grande número de variáveis em estudo, a eliminação daquelas que não são úteis na discriminação dos grupos.

Maroco (2003) sugere a utilização das estatísticas Distância de Mahalanobis, $V$ de Rao e Razão $F$ entre grupos para corroborar ou não a escolha efetuada com o uso do critério lambda de Wilks, e para diagnosticar possíveis problemas de multicolinearidade, o que implicaria a existência de viés no modelo, o autor indica a medida tolerância.

A Distância de Mahalanobis, $D M_{i j}$, entre variáveis $i$ e $j$, é dada por:

$$
D M_{i j}=\sqrt{\left(\left(X_{i}-X_{j}\right)^{\prime} S^{-1}\left(X_{i}-X_{j}\right)\right)}
$$


onde:

$X_{i}$ e $X_{j}=$ são variáveis dos grupos $i$ e $j$

$S^{-1}=$ Matriz de covariância

O quadrado dessa distância é utilizado para demonstrar quanto um grupo se diferencia de outro. Assim, uma variável é adicionada à função discriminante se a sua inclusão aumentar significativamente a $D M^{2}$. Essa estatística é sugerida quando as variáveis sob estudo apresentam correlações significativas que podem causar desvio no resultado da função discriminante.

O critério $V$ de Rao objetiva maximizar a distância entre os centróides dos grupos. Para tanto, é computado o valor do centróide geral pela fórmula:

$$
V=-(n-g) \sum_{i=1}^{p} \sum_{j=1}^{p} \mathrm{w}_{i j}{ }^{-1}\left(t_{i j}-w_{i j}\right),
$$

onde:

$n=$ dimensão da amostra

$g=$ número de grupos

$w_{i j}$ e $t_{i j}=$ elementos genéricos das matrizes $W$ e $T$, respectivamente.

A significância da variação do $V$ de Rao, derivada da adição ou remoção de uma variável, pode ser avaliada por aproximação à distribuição qui-quadrado com $g-1$ graus de liberdade.

A razão $F$ entre grupos é uma transformação da distância de Mahalanobis, considerando as diferentes dimensões entre os grupos, de maneira que os grupos de maior dimensão tenham maior peso na análise.

Dados dois grupos $a$ e $b$, a razão $F, F_{a b}$, é computada como:

$$
F_{a b}=\frac{(n-p-g) n_{a} n_{b}}{p(n-p)\left(n_{a}+n_{b}\right)} D M_{a b}^{2}
$$

onde:

$n=$ dimensão da amostra

$n_{a}=$ número de elementos do grupo a

$n_{b}=$ número de elementos do grupo b

$g=$ número de grupos

$p=$ número de variáveis

$D M_{a b}^{2}=$ Distância de Mahalanobis entre os grupos $a$ e $b$ 
A medida de tolerância de uma variável $X_{i}$ é definida como $T=1-R_{i}$, onde $R_{i}^{2}$ é o coeficiente de determinação do modelo composto da variável dependente, $X_{i}$, e as variáveis independentes, $X_{j}$. Quanto mais próximo de 0 (zero) é o valor da medida de tolerância, maior é o problema de correlação entre variáveis e mais instável será o modelo. Por outro lado, quanto mais próximo de 1 (um), mais estável será o modelo.

\section{ANÁLISE DOS RESULTADOS}

\subsection{Seleção das variáveis discriminantes (tabela 4)}

No nível de significância de $5,00 \%(\alpha=0,05)$ podemos inferir que somente as variáveis $\operatorname{LogAGRen}(p=0,111), \log \mathrm{ACir}(p=0,071), \operatorname{RORL}(p=0,084)$, EVARL $(p=0,160)$ e QROACir $(p=0,529)$ não são discriminantes.

Embora as variáveis LogAGRen e LogACir não tenham apresentado bons resultados, EstA, do mesmo subgrupo, correspondeu às expectativas. As variáveis RORL e QROACir, do subgrupo geração de caixa, não apresentaram resultados significantes, ao contrário de EBITCT, do mesmo grupo. Assim, os resultados não significativos de algumas variáveis podem ser explicados pela presença de outras variáveis, do mesmo subgrupo, que melhor explicam o problema estudado.

Tabela 4: Teste de igualdade das médias dos grupos

\begin{tabular}{lccccc}
\hline Variáveis & Lambda de Wilk & Estatística $\mathrm{F}$ & $d f_{1}$ & $d f_{2}$ & Sig. \\
\hline EstA & 0,630 & 40,0071450 & 1 & 68 & 0,000 \\
\hline LogAGRen & 0,963 & 2,6070666 & 1 & 68 & 0,111 \\
\hline LogACir & 0,953 & 3,3523506 & 1 & 68 & 0,071 \\
\hline EstC & 0,525 & 61,5461300 & 1 & 68 & 0,000 \\
\hline RCMCPd & 0,593 & 46,7486100 & 1 & 68 & 0,000 \\
\hline LogAFRLP & 0,912 & 6,5900086 & 1 & 68 & 0,012 \\
\hline EBITCT & 0,705 & 28,5128240 & 1 & 68 & 0,000 \\
\hline ReCP & 0,942 & 4,2170499 & 1 & 68 & 0,044 \\
\hline RORL & 0,957 & 3,0805090 & 1 & 68 & 0,084 \\
\hline Liq & 0,640 & 38,1943900 & 1 & 68 & 0,000 \\
\hline EVARL & 0,971 & 2,0157180 & 1 & 68 & 0,160 \\
\hline QROACir & 0,994 & 0,3997820 & 1 & 68 & 0,529 \\
\hline
\end{tabular}

Fonte: Elaborada pelos autores. 


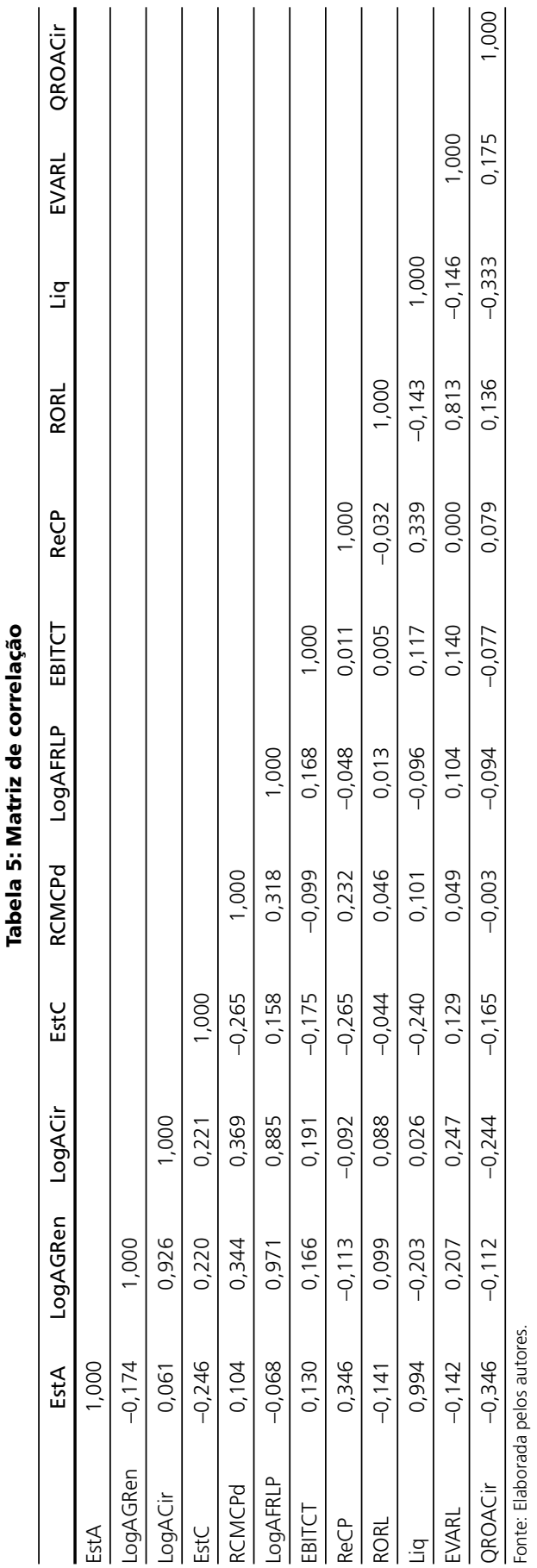




\subsection{Matriz de correlação (tabela 5)}

A matriz de correlação mostra que a variável LogACir tem forte correlação com as variáveis LogAGRen e LogAFRLP. Essa situação é derivada da composição das três variáveis, todas componentes do ativo total. As variáveis Liq e EstA também apresentaram correlação significativa, assim como EVARL e RORL. As duas últimas têm em sua composição as receitas líquidas, o que provavelmente determinou a correlação.

\subsection{Análise conjunta dos testes realizados (tabela 6)}

A tabela 6 mostra que as variáveis que não apresentaram o sinal esperado também mostraram problemas de significância, correlação ou normalidade. Nenhum tratamento de correção foi utilizado, primeiramente para que fosse mantida a generalidade do modelo e também porque as variáveis selecionadas pelo método passo a passo cumpriram os pressupostos da análise discriminante.

Tabela 6: Resumo dos testes realizados

\begin{tabular}{lccccccc}
\hline Referência & Variável & $\begin{array}{c}\text { Ausência } \\
\text { de outliers }\end{array}$ & Normalidade & Homoced. & $\begin{array}{c}\text { Ausência } \\
\text { de correlação }\end{array}$ & $\begin{array}{c}\text { Sinal } \\
\text { esperado Significância }\end{array}$ \\
\hline Estrutura & EstA & não & $\operatorname{sim}$ & $\operatorname{sim}$ & não & $\operatorname{sim}$ & $\operatorname{sim}$ \\
de & LogAGRen & $\operatorname{sim}$ & $\operatorname{sim}$ & $\operatorname{sim}$ & não & $\operatorname{sim}$ & não \\
ativos & LogACir & não & $\operatorname{sim}$ & $\operatorname{sim}$ & não & não & não \\
\hline Estrutura & EstC & não & $\operatorname{sim}$ & $\operatorname{sim}$ & $\operatorname{sim}$ & $\operatorname{sim}$ & $\operatorname{sim}$ \\
de & RCMCPd & não & $\operatorname{sim}$ & $\operatorname{sim}$ & $\operatorname{sim}$ & $\operatorname{sim}$ & $\operatorname{sim}$ \\
capital & LogAFRLP & $\operatorname{sim}$ & $\operatorname{sim}$ & $\operatorname{sim}$ & não & $\operatorname{sim}$ & $\operatorname{sim}$ \\
\hline Geração & EBITCT & não & $\operatorname{sim}$ & $\operatorname{sim}$ & $\operatorname{sim}$ & $\operatorname{sim}$ & $\operatorname{sim}$ \\
de & ReCP & não & não & $\operatorname{sim}$ & $\operatorname{sim}$ & não & $\operatorname{sim}$ \\
caixa & RORL & não & não & não & não & $\operatorname{sim}$ & não \\
& Liq & não & $\operatorname{sim}$ & $\operatorname{sim}$ & não & não & sim \\
& QROACir & não & não & $\operatorname{sim}$ & sim & não & não \\
\hline Eficiência & EVARL & não & não & $\operatorname{sim}$ & não & sim & não \\
\hline
\end{tabular}

Fonte: Elaborada pelos autores.

Tabela 7: Teste de homogeneidade

\begin{tabular}{lcc}
\hline M de Box & & 674,297 \\
\hline $\mathbf{F}$ & Aproximado & 7,019 \\
\hline & $\mathrm{df1}$ & 78,000 \\
\hline $\mathrm{df2}$ & $14.601,821$ \\
\hline & Sig. & 0,000 \\
\hline
\end{tabular}




\subsection{Estimação da função discriminante pelo método direto}

O resultado do teste $M$ de Box, tabela 7, determinou a rejeição da hipótese de serem iguais as matrizes de variâncias-covariâncias entre os grupos (heterocedasticidade). Assim, a função derivada pelo método direto foi invalidada, posto que houve violação de um dos principais pressupostos da análise discriminante.

\subsection{Estimação da função discriminante pelo método passo a passo}

A estimação pelo método passo a passo apresentou os seguintes resultados, com os quais definimos a seguinte função discriminante:

$$
\begin{aligned}
& y=0,526+1,6791 E s t A-1,8309 E s t C+0,012 R C M C P d+2,0812 \text { EBICT } \\
& \text { E o ponto de corte } Y=\frac{(35 \times-1,333)+(35 \times 1,333)}{(35+35)}=0
\end{aligned}
$$

A partir desses resultados, podemos dizer que o postulante a crédito será classificado no grupo 0 (provável insolvente), se $Y<0$, e no grupo 1 (provável solvente), se $Y>0$.

Embora a variável LogAFRLP tenha apresentado nível de significância satisfatório, ela não foi incluída na função discriminante derivada pelo método passo a passo. A exclusão dessa variável deve-se ao fato de que outras

Tabela 8: Sumário dos casos utilizados na análise

\begin{tabular}{lccc}
\hline Grupos & Percentual & Casos analisados & Centróides \\
\hline 0 & 50 & 35 & $-1,333$ \\
\hline 1 & 50 & 35 & 1,333 \\
\hline Fonte: Elaborada pelos autores. & &
\end{tabular}

Tabela 9: Coeficientes da função discriminante

\begin{tabular}{lccc}
\hline Variáveis & Coeficientes & Sinal esperado & Sinal calculado \\
\hline EstA & 1,6791 & Positivo & Positivo \\
\hline EstC & $-1,8309$ & Negativo & Negativo \\
\hline RCMCPd & 0,0120 & Positivo & Positivo \\
\hline EBITCT & 2,0812 & Positivo & Positivo \\
\hline Constante & 0,526 & & \\
\hline
\end{tabular}

Fonte: Elaborada pelos autores. 
Tabela 10: Teste de tolerância

\begin{tabular}{ccccc}
\hline & Passo & Tolerância & F mínimo & Lambda de Wilks \\
\hline 1 & ESTC & 1,000 & 61,546 & \\
\hline \multirow{2}{*}{ ESTC } & 0,930 & 22,852 & 0,593 \\
& RCMCPD & 0,930 & 12,589 & 0,525 \\
\hline \multirow{4}{*}{3} & ESTC & 0,889 & 11,353 & 0,450 \\
& RCMCPD & 0,908 & 14,276 & 0,467 \\
& EBITCT & 0,947 & 10,037 & 0,442 \\
\hline \multirow{4}{*}{4} & ESTC & 0,852 & 6,560 & 0,389 \\
& RCMCPD & 0,905 & 11,550 & 0,416 \\
& EBITCT & 0,937 & 7,390 & 0,394 \\
& ESTA & 0,929 & 5,500 & 0,384 \\
\hline
\end{tabular}

Fonte: Elaborada pelos autores.

Tabela 11: Matriz de classificação, método passo a passo

\begin{tabular}{lccccc}
\hline Situação & Grupos & $\begin{array}{c}\text { Classificados } \\
\text { a priori }\end{array}$ & Previstos & $\begin{array}{c}\text { Classificação } \\
\text { correta (\%) }\end{array}$ & $\begin{array}{c}\text { Acerto } \\
\text { total (\%) }\end{array}$ \\
\hline Original & 0 & 35 & 31 & 88,60 & 88,60 \\
\hline $\begin{array}{l}\text { Validação } \\
\text { cruzada }\end{array}$ & 1 & 35 & 31 & 88,60 & 88,60 \\
\hline
\end{tabular}

Fonte: Elaborada pelos autores.

Tabela 12: Teste de homogeneidade

\begin{tabular}{lcc}
\hline$M$ de Box & & 18,848 \\
\hline$F$ & Aproximado & 1,765 \\
\hline$d f 1$ & 10,000 \\
\hline$d f 2$ & $22.106,773$ \\
\hline Sig. & 0,061 \\
\hline
\end{tabular}

Fonte: Elaborada pelos autores.

variáveis do modelo têm poder explicativo suficiente sobre o problema estudado. A utilização de outros critérios estatísticos (Distância de Mahalanobis, $V$ de Rao e Razão $F$ ), para validar a função computada pelo método passo a passo, corrobora a escolha feita pelo critério lambda de Wilks, e a possibilidade de existirem problemas derivados de multicolinearidade foi afastada, com o diagnóstico efetuado através da medida de tolerância que apresentou os resultados seguintes, conforme a tabela 10. 
Quanto mais próximo de 1 (um), maior é a independência de uma variável em relação às demais, enquanto um resultado 0 (zero) mostra o oposto.

A função computada pelo método alternativo classificou corretamente $88,6 \%$ dos casos, conforme a tabela 11 .

O teste $M$ de Box com p-valor 0,061, comparado ao nível de significância de 5,0\% $(\alpha=0,05)$, permite não rejeitar a hipótese $\left(H_{0}\right)$ de igualdade das matrizes de variância-covariância (homocedasticidade) para os dois grupos. Deve-se observar que, apesar de relativamente baixo, o teste $M$ de Box é muito sensível ao tamanho da amostra, sendo, por essa razão, aceitável o resultado encontrado (Hair Jr. et al., 2005, p. 236).

\subsection{Normalidade univariada e multivariada}

As tabelas 13 e 14 mostram os resultados dos testes de normalidade das variáveis. Com todos os $p$-valor - Asymp. Sig (2-tailed), na tabela 13, e $p$ valor, na tabela 14 - maiores que o nível de significância de 5,0\% ( $\alpha=$ $0,05)$, podemos aceitar tanto a hipótese de normalidade individual quanto a de normalidade conjunta das variáveis da função derivada pelo método passo a passo.

Tabela 13: Normalidade univariada

\begin{tabular}{llrrrr}
\hline & Variáveis & \multicolumn{1}{c}{ ESTA } & ESTC & RCMCPD & EBITCT \\
\hline Parâmetros & Média & 70 & 70 & 70 & 70 \\
normais & Desvio-padrão & $-0,0190$ & 0,4863 & 20,1774 & 0,0741 \\
\hline Maiores & 0,2688 & 0,3056 & 54,6512 & 0,2333 \\
diferenças & Absoluta & 0,0708 & 0,1048 & 0,0725 & 0,1014 \\
& Positiva & 0,0463 & 0,0942 & 0,0684 & 0,1014 \\
& Negativa & $-0,0708$ & $-0,1048$ & $-0,0725$ & $-0,0718$ \\
\hline & Kolmogorov-Smirnov Z & 0,5924 & 0,8767 & 0,6069 & 0,8482 \\
\hline
\end{tabular}

Fonte: Elaborada pelos autores

Tabela 14: Normalidade multivariada

\begin{tabular}{lccc}
\hline Teste & Coeficientes & Estatística & $p$-valor \\
\hline Mardia-Assimetria & 2,53 & 31,312 & 0,051 \\
\hline Mardia-Curtose & 24,453 & 0,274 & 0,784 \\
\hline Henze-Zirkler & & 0,915 & 0,228 \\
\hline
\end{tabular}

Fonte: Elaborada pelos autores. 
Tabela 15: Teste de significância da função

\begin{tabular}{lcccc}
\hline Função & Autovalor & \% Variância & Total & Correlação canônica \\
\hline 1 & 1,828 & 100 & 100 & 0,804 \\
\hline Fonte: Elaborada pelos autores. & & &
\end{tabular}

Tabela 16: Teste de significância da função

\begin{tabular}{lcccc}
\hline Função & Lambda de Wilks & Qui-quadrado & df & Sig. \\
\hline 1 & 0,354 & 68,616 & 4 & 0,000 \\
\hline Fonte: Elaborada pelos autores. & & &
\end{tabular}

\subsection{Significância da função discriminante}

Com a existência de dois grupos em estudo, somente uma função foi estimada. O autovalor (medida relativa de quão diferentes são os grupos sob estudo) associado a essa função foi 1,828, que responde a $100 \%$ da variância explicada. A correlação canônica associada à função - que mede o alcance da associação entre os escores discriminantes e os grupos - foi 0,804, que, elevada ao quadrado, indica que $64 \%$ da variável dependente são explicados pelo modelo. Com o resultado de 0,354 para o lambda de Wilks equivalente ao qui-quadrado de 68,616 e p-valor 0,000, podemos inferir que a função é altamente significativa.

\section{CONCLUSÃO}

A construção de um modelo de previsão de insolvência com utilização da análise discriminante e dados contábeis foi o objetivo principal deste estudo. O modelo proposto, derivado pelo método passo a passo, apresentou índice de acerto de $88,6 \%$, valor superior a resultados de alguns modelos construídos no passado com a mesma técnica.

A inclusão de empresas com patrimônio líquido negativo no grupo das empresas ruins foi uma abordagem diferenciada em relação a trabalhos anteriores, que consideravam como empresas insolventes somente aquelas com concordata ou falência decretada.

Os testes de normalidade (Kolmogorov-Smirnov, Mardia-Assimetria, Mardia-Curtose e Henze-Zirkler), a existência de igualdade das matrizes de variância-covariância dos grupos conferida pelo teste $M$ de Box e a ausência de multicolinearidade garantida pela medida de tolerância indicaram que as 
variáveis preditoras, selecionadas para compor a função discriminante pelo método passo a passo, cumpriram os principais pressupostos da análise discriminante e garantiram a robustez do modelo proposto.

Com relação às variáveis independentes — EstA, Estc, EBITCT e RCMCPd —, o estudo confirmou o poder discriminatório das primeiras três variáveis, como nos trabalhos de Lo (1986), Sanvicente e Minardi (1998), Glen (2004) e Silva (2003). Essas variáveis evidenciam, respectivamente, as decisões financeiras sobre estrutura de ativos, estrutura de capital e a geração de caixa.

A variável RCMCPd, representativa do montante dos recursos próprios disponíveis, também apresentou resultados satisfatórios. Essa variável é utilizada normalmente para avaliar se o montante de capital próprio das instituições financeiras é suficiente para a cobertura dos riscos assumidos por aquelas entidades. $\mathrm{O}$ resultado conseguido no presente trabalho com empresas não financeiras mostrou que a variável tem poder discriminante $\mathrm{e}$ pode ser usada como variável preditora da probabilidade de insolvência de firmas não financeiras.

Em resumo, podemos afirmar, com base nos resultados apresentados, que o modelo proposto tem robustez estatística, representa, através das suas variáveis independentes, o resultado das decisões das firmas e tem bom índice de acerto nas predições, o que o credencia como ferramenta importante na gestão de risco de crédito das empresas.

\section{NOTAS}

1. Classificação.

2. Impossibilidade de pagamento, insolvência.

\section{REFERÊNCIAS BIBLIOGRÁFICAS}

BACK, B. et al. Choosing bankruptcy predictors using discriminant analysis, logit analysis, and genetic algorithms. Turku Centre for Computer Science Technical Report, n. 40, ISBN 951-650-828-6, ISSN 1239-1891, p. 1-5, sep. 1996.

BARTH, N. L. Inadimplência: construção de modelos de previsão. São Paulo: Nobel, 2004. p. 3, 10-15 e 21-30,.

BUSSAB, W. de O.; MORETTIN, P. A. Estatística básica. 5. ed. São Paulo: Saraiva, 2003. p. 343. 
CAOUETTE, J. B.; ALTMAN, E. I.; NARAYANAM, P. Gestão do risco de crédito: o próximo grande desafio do futuro. Tradução de Allan Hastings. Supervisão técnica João Carlos Douat. Rio de Janeiro: Qualitymark, 1999. p. 119-121 e 130-144.

GLEN, J. Debit and firm vulnerability, 2004. Disponível em http://www.defaultrisk.com/ps_ scoring.htm. Acesso: 21 set. 10:50, p. 6-8, 2005.

HAIR JR.; JOSEPH, F.; ANDERSON, R. E.; TATHAM, R. L.; BLACK, W. Análise multivariada de dados. Tradução Adonai Schlup Santánna e Anselmo Chaves Neto. Supervisão técnica Maria Aparecida Gouvêa. 5. ed. Porto Alegre: Bookman, 2005. p. 80-87 e 209-230.

HARRIS, M.; RAVIV, A. The theory of capital structure. The Journal of Finance, v. 46. n. 1, p. 297-355, mar. 1991.

LO, A. W. Logit versus discriminant analisys: a specification test with application to corporate bankruptcies. Journal of Econometrics, v. 31, p. 151-178, 1986.

MAROCO, J. Análise estatística com utilização do SPSS. 2. ed. Lisboa: Silabo, 2003. p. 56-59, $112-114$ e 332-360.

MECKLIN, C. J.; MUNDFROM, D. J. On using asymptotic critical values in testing for multivariate normality. Disponível em http://interstat.statjournals.net/YEAR/2003/articles/0301001.pdf. Acesso: 4 fev., 11:30, p. 1-12, 2008.

REQUIÃO, R. Curso de direito falimentar. 17. ed. São Paulo: Saraiva, 1998. p. 56.

SANVICENTE, A. Z.; MINARDI, A. M. A. F. Identificação de indicadores contábeis significativos para previsão de concordata de empresas, 1998. Disponível em: www.risktech.com.br/ PDFs/indicadores_concordata.pdf. Acesso: 15 ago. 09:21, p. 1-3, 2005.

SHARMA, S. Applied Multivariate Techniques. 1. ed. University os South Carolina, 1996. p. 263-264.

SILVA, J. P. da. Gestão e análise de risco de crédito. 4. ed. São Paulo: Atlas, 2003. p. 63. 